\title{
Probing the gluon tomography in photoproduction of dipion
}

\author{
Yoshikazu Hagiwara, Cheng Zhang, Jian Zhou®, and Ya-jin Zhou \\ Key Laboratory of Particle Physics and Particle Irradiation (MOE), Institute of Frontier and \\ Interdisciplinary Science, Shandong University, (QingDao), Shandong 266237, China
}

(Received 11 July 2021; accepted 13 October 2021; published 17 November 2021)

\begin{abstract}
A sizable $\cos 4 \phi$ azimuthal asymmetry in exclusive dipion production near $\rho^{0}$ resonance peak in ultraperipheral heavy-ion collisions recently has been reported by STAR collaboration. We show that both elliptic gluon Wigner distribution and final-state soft photon radiation can give rise to this azimuthal asymmetry. The fact that the QED effect alone severely underestimates the observed asymmetry might signal the existence of the nontrivial correlation in quantum phase distribution of gluons.
\end{abstract}

DOI: 10.1103/PhysRevD.104.094021

\section{INTRODUCTION}

The study of nucleon/nucleus multiple-dimensional partonic structure has been widely recognized as one of the most important frontiers of hadronic physics. Within perturbative QCD factorization framework, 3D partonic structure of nucleon is quantified by transverse momentumdependent distributions (TMDs) and generalized parton distributions (GPDs). The mother distribution of these two, namely quantum phase space Wigner distribution, later was introduced [1] to accommodate more comprehensive information on nucleon internal structure, such as parton canonical orbital angular momentum [2-5], the quantum correlation between the position and momentum of partons. The Fourier transform of the Wigner distribution known as the generalized TMD distributions (GTMDs) [6,7] is also often used in phenomenological studies. Pioneered by a recent work [8], a few proposals for extracting the Wigner distribution in hard scattering processes in ep/A collisions for gluon sector [8-19] and pp collisions for quark sector [20] have been put forward.

One special gluon Wigner distribution commonly referred to as the elliptic gluon Wigner distribution [8] has recently attracted much attention [9-18]. It describes an azimuthal correlation between the impact parameter and gluon transverse momentum. In the small- $x$ limit, this gluon distribution can be dynamically generated either from a semiclassical model or from small- $x$ evolution. In the kinematic region where nuclear recoil transverse momentum and gluon transverse momentum are comparable, the MV model calculation predicates a rather sizable

Published by the American Physical Society under the terms of the Creative Commons Attribution 4.0 International license. Further distribution of this work must maintain attribution to the author(s) and the published article's title, journal citation, and DOI. Funded by SCOAP ${ }^{3}$. correlation [17], which we view as a very encouraging result. This distribution is proposed to be extracted via a $\cos 2 \phi$ azimuthal asymmetry in diffractive dijet production in ep/A collisions. To avoid the contributions from the final-state soft gluon radiation to the asymmetry [21-25], it is necessary to directly measure nuclear recoil transverse momentum instead of the total transverse momentum of dijet, which imposes a big challenge to experimental measurement.

On the other hand, among many exciting topics [26-34], ultraperipheral heavy-ion collisions(UPCs) provide us unique chances to investigate partonic structure of nucleus before the advent of the Electron Ion Collider (EIC) era. In particular, with the discovery of linear polarization of coherent photons [35-39], a new experimental avenue is opened to study nucleus structure via the novel polarization-dependent observables in photonuclear reactions, which already led to fruitful results [40-43]. By coupling with elliptic gluon distribution, linearly polarized coherent photon distribution also plays an important role in inducing the $\cos 4 \phi$ asymmetry in exclusive $\pi^{+} \pi^{-}$pair production in ultraperipheral heavy-ion collisions recently reported by STAR collaboration [39], where $\phi$ is the azimuthal angle between pion's transverse momentum and the pion pair's total transverse momentum.

To be more specific, the $\cos 4 \phi$ asymmetry partially results from an interference contribution. Near the $\rho^{0}$ resonance peak, the dipion is dominantly from the decay of $\rho^{0}$ which is produced in the coherent photonuclear reaction. However, there is also a non-negligible contribution to the pair-invariant mass spectrum from the interference contributions between direct pions production and those from the $\rho^{0}$ decay [43-48]. Such an interference effect not only leads to sizable deviation from the BreitWigner resonance shape [44], but also generates the mentioned $\cos 4 \phi$ azimuthal modulation. The underlying physics of the $\cos 4 \phi$ modulation from the interference 
effect is that the orbital angular momentum carried by dipion in the direct production amplitude and in the conjugate $\rho^{0}$ decay amplitude differ by four units angular momentum. In the next section, we explain in details how this mechanism is realized through the coupling of elliptic gluon distribution and linearly polarized photon distribution. Though the azimuthal asymmetry can result from the final-state soft photon radiation effect [23] as well, our numerical results suggest that the QED effect alone is not sufficient to account for the observed asymmetry.

The paper is structured as follows. We compute the contributions to the asymmetry from both elliptic gluon distribution and final-state soft photon radiation in the next section, followed by the numerical results presented in Sec. III. The paper is summarized in Sec. IV.

\section{II. $\cos 4 \phi$ AZIMUTHAL ASYMMETRY IN EXCLUSIVE PION PAIR PRODUCTION}

We start by briefly reviewing the calculation of polarization-independent diffractive $\rho^{0}$ production (for the recent experimental studies of the process, see Refs. [49-51]). It is conventionally formulated in the dipole model $[52,53]$, where the whole process is divided into three steps: quasireal photon splitting into quark and antiquark pair, the color dipole scattering off nucleus, and subsequently recombining to form a vector meson after penetrating the nucleus target. Following this picture, one can directly write the $\rho^{0}$ production amplitude (see Refs. [40,43] for more details),

$\mathcal{A}\left(x_{g}, \Delta_{\perp}\right)=\int d^{2} b_{\perp} e^{-i \Delta_{\perp} \cdot b_{\perp}} \int \frac{d^{2} r_{\perp}}{4 \pi} N\left(r_{\perp}, b_{\perp}\right)\left[\Omega^{*} K\right]\left(r_{\perp}\right)$,

where $N\left(r_{\perp}, b_{\perp}\right)$ is the dipole-nucleus scattering amplitude, and $\Delta_{\perp}$ is nuclear recoil transverse momentum. And, $\left[\Omega^{*} K\right]$ denotes the overlap of photon wave function and the vector meson wave function,

$$
\begin{aligned}
{\left[\Omega^{*} K\right]\left(r_{\perp}\right)=} & \frac{N_{c} e e_{q}}{\pi} \int_{0}^{1} d z\left\{m_{q}^{2} \Omega^{*}\left(\left|r_{\perp}\right|, z\right) K_{0}\left(\left|r_{\perp}\right| e_{f}\right)\right. \\
& \left.+\left[z^{2}+(1-z)^{2}\right] \frac{\partial \Omega^{*}\left(\left|r_{\perp}\right|, z\right)}{\partial\left|r_{\perp}\right|} \frac{\partial K_{0}\left(\left|r_{\perp}\right| e_{f}\right)}{\partial\left|r_{\perp}\right|}\right\} \\
& \times e^{i\left(z-\frac{1}{2}\right) \Delta_{\perp} \cdot r_{\perp},}
\end{aligned}
$$

where $z$ stands for the fraction of photon's light-cone momentum carried by quark. Quark and antiquark helicities and color sums have been performed in the above formula. $K_{0}$ is a modified Bessel function of the second kind. $\Omega$ is the scalar part of vector meson wave function. Note that a phase factor $e^{i\left(z-\frac{1}{2}\right) \Delta_{\perp} \cdot r_{\perp}}$ is included to account for the nonforward correction $[14,54,55]$.
One can easily derive the dipion production amplitude by multiplying the $\rho^{0}$ production amplitude with a simplified Breit-Wigner form which describes the transition from $\rho^{0}$ to $\pi^{+} \pi^{-}$,

$$
\mathcal{M}_{r}=i \mathcal{A}\left(x_{g}, \Delta_{\perp}\right) \frac{f_{\rho \pi \pi} P_{\perp} \cdot \hat{k}_{\perp}}{Q^{2}-M_{\rho}^{2}+i M_{\rho} \Gamma_{\rho}},
$$

where $M_{\rho}$ is $\rho^{0}$ mass. $Q$ is the invariant mass of the dipion system and $P_{\perp}$ is defined as $P_{\perp}=\left(p_{1 \perp}-p_{2 \perp}\right) / 2$, with $p_{1 \perp}$ and $p_{2 \perp}$ being the produced pions' transverse momenta. The polarization vector of the incident photon is replaced with its transverse momentum $k_{\perp}$ by making use of the fact that the coherent photons are naturally linearly polarized. $f_{\rho \pi \pi}$ is the effective coupling constant and fixed to be $f_{\rho \pi \pi}=12.24$ according to the optical theorem with the parameter $\Gamma_{\rho}=0.156 \mathrm{GeV}$. With this production amplitude, it is straightforward to obtain the polarizationindependent cross section $\frac{d \sigma}{d \mathcal{P} . \mathcal{S} \text {, }}$, where $\mathcal{P}$.S. represents the phase space.

The mentioned $\cos 4 \phi$ azimuthal asymmetry can be induced by soft photon radiation from the final-state charged pion particles. The corresponding physics from the finalstate photon radiation is captured by the soft factor which enters the differential cross-section formula via [21-24],

$$
\frac{d \sigma\left(q_{\perp}\right)}{d \mathcal{P} . \mathcal{S} .}=\int d^{2} q_{\perp}^{\prime} \frac{d \sigma_{0}\left(q_{\perp}^{\prime}\right)}{d \mathcal{P} . \mathcal{S} .} S\left(q_{\perp}-q_{\perp}^{\prime}\right)
$$

where $\sigma_{0}$ is the leading-order Born cross section whose full expression can be found in Refs. $[40,43]$. The soft factor is expanded at the leading order as [24]

$S\left(l_{\perp}\right)=\delta\left(l_{\perp}\right)+\frac{\alpha_{e}}{\pi^{2} l_{\perp}^{2}}\left\{c_{0}+2 c_{2} \cos 2 \phi+2 c_{4} \cos 4 \phi+\cdots\right\}$,

where $\phi$ is the angle between $P_{\perp}$ and soft photon transverse momentum $-l_{\perp}$. When the final-state particle mass is much smaller than $P_{\perp}$, there exists the analytical expressions for the coefficients $c_{0} \approx \ln \frac{Q^{2}}{m_{\pi}^{2}}, \quad c_{2} \approx \ln \frac{Q^{2}}{m_{\pi}^{2}}+\delta y \sinh \delta y-$ $2 \cosh ^{2} \frac{\delta y}{2} \ln [2(1+\cosh \delta y)] \ldots$, where $Q$ is the invariant mass of the dipion system and $\delta y=y_{1}-y_{2}$ is the difference between two pions' rapidities. For the current case, the mass of the final-state charged particles is the same order of the invariant mass. We thus proceed numerically and obtain $c_{0} \approx 3.5, c_{2} \approx 1.1$, and $c_{4} \approx 0.42$. The rapidity dependence of these coefficients is quite mild for Relativistic Heavy Ion Collider (RHIC) kinematics and is neglected.

Following the standard procedure, the soft factor in Eq. (5) can be extended to all orders by exponentiating the azimuthal independent part to the Sudakov factor in the transverse position space. The resummed cross section takes the form [21-24] 


$$
\begin{aligned}
\frac{d \sigma\left(q_{\perp}\right)}{d \mathcal{P} . \mathcal{S} .}= & \int \frac{d^{2} r_{\perp}}{(2 \pi)^{2}}\left[1-\frac{2 \alpha_{e} c_{2}}{\pi} \cos 2 \phi_{r}+\frac{\alpha_{e} c_{4}}{\pi} \cos 4 \phi_{r}\right] \\
& \times e^{i r_{\perp} \cdot q_{\perp}} e^{-\operatorname{Sud}\left(r_{\perp}\right)} \int d^{2} q_{\perp}^{\prime} e^{i r_{\perp} \cdot q_{\perp}^{\prime}} \frac{d \sigma\left(q_{\perp}^{\prime}\right)}{d \mathcal{P} . \mathcal{S} .}
\end{aligned}
$$

Here $\phi_{r}$ is the angle between $r_{\perp}$ and $P_{\perp}$. In the following numerical estimation, we took into account power corrections from the soft factor following the method outlined in Ref. [24]. The Sudakov factor at one loop is given by [24]

$$
\operatorname{Sud}\left(r_{\perp}\right)=\frac{\alpha_{e}}{\pi} c_{0} \ln \frac{P_{\perp}^{2}}{\mu_{r}^{2}}
$$

with $\mu_{r}=2 e^{-\gamma_{E}} /\left|r_{\perp}\right|$. The Sudakov factor plays a critical role in yielding perturbative high $-q_{\perp}$ tail of lepton pair produced in UPCs $[37,56]$.

We now turn to derive the direct dipion production amplitude. In the collinear factorization, the transition from quark-antiquark pair to dipion pair is described by the nonperturbative and universal dipion distribution amplitude (DA) [57-59]. For the current case, it is necessary to go beyond the collinear factorization and take into account quark and antiquark finite transverse momenta (or transverse position) effects in order to preserve the information of the orbital angular momentum, which is essential for yielding the nontrivial azimuthal modulation. To this end, we assume that the transverse position of $\pi^{+}$is the same as the leading $u$ or $\bar{d}$ quark during this nonperturbative transition, though the longitudinal momentum is redistributed during the transition. The similar requirement applies to $\pi^{-}$as well. We notice that our assumption for the transverse spatial distribution differs from the analysis made in Ref. [60]. This difference might be attributed to the fact that quark and antiquark transverse momenta have been integrated out in the work [60], while transverse momenta of quark and the corresponding pion are implicitly assumed to be the same in our treatment. By making this crude approximation, the amplitude reads

$$
\begin{aligned}
\mathcal{M}_{d}= & i \int d^{2} b_{\perp} e^{i \Delta_{\perp} \cdot b_{\perp}} \int \frac{d^{2} r_{\perp}}{4 \pi} \int d z \\
& \times \Psi^{\gamma \rightarrow q \bar{q}}\left(r_{\perp}, z, \epsilon_{\perp \gamma}\right) N\left(r_{\perp}, b_{\perp}\right) \Phi(z, \zeta) e^{i r_{\perp} \cdot P_{\perp}},
\end{aligned}
$$

where $\Phi$ is a universal nonperturbative function describing the transition from a quark-antiquark pair to a dipion system. $\zeta$ is the longitudinal momentum fraction carried by $\pi^{+}$. We stress that any nonperturbative model for the quark-pair to pion-pair transition would generate a $\cos 4 \phi$ azimuthal asymmetry, as long as it conserves angular momentum.

We proceed to explicitly work out the angular structure residing in $\mathcal{M}_{d}$ by first writing the polarization-dependent photon wave function,

$$
\Psi^{\gamma \rightarrow q \bar{q}}\left(r_{\perp}, z, \epsilon_{\perp}^{\gamma}\right)=\frac{e e_{q}}{2 \pi} \delta_{i j}\left\{\delta_{\sigma \sigma^{\prime}} m_{q}\left(\epsilon_{\perp}^{\gamma, 1}+i \sigma \epsilon_{\perp}^{\gamma, 2}\right)-\delta_{\sigma,-\sigma^{\prime}}\left[(1-2 z) i \epsilon_{\perp}^{\gamma} \cdot \hat{r}_{\perp}+\sigma \epsilon_{\perp}^{\gamma} \times \hat{r}_{\perp}\right] \frac{\partial}{\partial\left|r_{\perp}\right|}\right\} K_{0}\left(\left|r_{\perp}\right| e_{f}\right),
$$

where $\sigma$ and $\sigma^{\prime}$ are the quark and antiquark helicities, $i, j$ are the color indices. $\epsilon_{\perp}^{\gamma}=\hat{k}_{\perp} \equiv k_{\perp}\left|k_{\perp}\right|$ as explained above. $m_{q}$ and $e_{q}$ denote quark mass and quark's electric charge number with flavor $q$, respectively. $e_{f}$ is defined as $e_{f}^{2}=Q^{2} z(1-z)+m_{q}^{2}$ for the virtual photon case. For the quasireal photon case, $e_{f}=140 \mathrm{MeV}$ is determined by fitting to Hadron-Electron Ring Accelerator data.

We proceed to discuss the symmetric properties of $\Phi(z, \zeta)$ in order to have some guidance for parametrizing this nonperturbative function. The invariance of the scattering amplitude under the charge-parity transformation requires

$$
\Phi(z, \zeta)=-\Phi(1-z, 1-\zeta) .
$$

On the other hand, the final-state produced dipion is a $C$-odd state since the orbital angular momentum quantum number carried by dipion is either 1 or 3 in the current case. This implies that

$$
\Phi(z, \zeta) e^{i r_{\perp} \cdot P_{\perp}}=-\Phi(z, 1-\zeta) e^{-i r_{\perp} \cdot P_{\perp}} .
$$

Using these relations, one obtains

$$
\begin{aligned}
\Phi(z, \zeta) e^{i r_{\perp} \cdot P_{\perp}}= & \frac{\Phi(z, \zeta)+\Phi(z, 1-\zeta)}{2} e^{i r_{\perp} \cdot P_{\perp}} \\
& -\Phi(z, 1-\zeta) \frac{e^{-i r_{\perp} \cdot P_{\perp}}+e^{i r_{\perp} \cdot P_{\perp}}}{2}
\end{aligned}
$$

where the contribution in the second line decouples after convoluting with the photon wave function and the dipole amplitude in Eq. (8). More discussions on the symmetric properties for the integrated dipion DA can be found in Ref. [61]. We are then motivated to parametrize the $z$ integration in Eq. (8) as the following:

$$
\begin{aligned}
\int d z(1-2 z) \Phi(z, \zeta) & =\int d z(1-2 z) \frac{\Phi(z, \zeta)+\Phi(z, 1-\zeta)}{2} \\
& =\delta_{\sigma,-\sigma^{\prime}} \delta^{i j} \zeta(1-\zeta) \mathcal{C},
\end{aligned}
$$

where $\zeta(1-\zeta)$ is a commonly used parametrization that respects particle-antiparticle symmetry. The constant $\mathcal{C}$ is determined by fitting to the polarization-averaged cross 
section which receives the sizable contribution from the interference term. We close the discussion about dipion distribution amplitude with a final remark. $\Phi(z, \zeta)$ does not correspond to the normal leading-twist collinear dipion distribution amplitude. Actually, the polarizationindependent leading-twist dipion DA decouples from the process under consideration once averaging over the polarizations of the incident photon. Instead, the high-twist collinear DAs that contribute to the observable are related to the functions $\left.\frac{\partial}{\partial r_{\perp}^{i}} \Phi(z, \zeta) e^{i r_{\perp} \cdot P_{\perp}}\right|_{r_{\perp} \rightarrow 0}$ for the $p$-wave final state and $\left.\frac{\partial}{\partial r_{\perp}^{i}} \frac{\partial}{\partial r_{\perp}^{j}} \frac{\partial}{\partial r_{\perp}^{k}} \Phi(z, \zeta) e^{i r_{\perp} \cdot P_{\perp}}\right|_{r_{\perp} \rightarrow 0}$ for the $f$-wave final state. Here we refrain ourselves from giving the explicit matrix element definition for these dipion DAs as we do not attempt to extensively discuss the properties of high-twist dipion DAs in this work.

We now study the angular correlation in the dipole amplitude $N\left(b_{\perp}, r_{\perp}\right)$, which reads in the MV model $[62,63]$

$N\left(b_{\perp}, r_{\perp}\right)=1-e^{-\alpha_{s} C_{F} \frac{1}{8 \pi} \int d^{2} x_{\perp} \mu_{A}\left(x_{\perp}\right)\left[\ln \frac{\left(b_{\perp}+\frac{r_{\perp}}{r_{\perp}}-x_{\perp}\right)^{2}}{\left(b_{\perp}-\frac{-1}{2}-x_{\perp}\right)^{2}}\right]^{2}}$,

where $\mu_{A}\left(x_{\perp}\right)$ is the color source distribution. If $\mu_{A}\left(x_{\perp}\right)$ is not uniformly distributed in the transverse plane of nucleus, a nontrivial angular correlation between $b_{\perp}$ and $r_{\perp}$ can arise. By Taylor expanding the above expression and ignoring high-order harmonics, one obtains

$$
\begin{aligned}
N\left(b_{\perp}, r_{\perp}\right) \approx & 1-\exp \left[-Q_{s}^{2}\left(b_{\perp}^{2}\right) r_{\perp}^{2} / 4\right] \ldots \ldots \\
& +E\left(b_{\perp}^{2}, r_{\perp}^{2}\right) 2 \cos \left(2 \phi_{b}-2 \phi_{r}\right) \\
& \times \frac{Q_{s}^{2}\left(b_{\perp}^{2}\right) r_{\perp}^{2}}{4} e^{-\frac{Q_{s}^{2}\left(b_{\perp}^{2}\right) r_{\perp}^{2}}{4}}
\end{aligned}
$$

The coefficient $E\left(b_{\perp}^{2}, r_{\perp}^{2}\right)$ has been explicitly computed in the MV model for different kinematic regions $[10,12,16,17]$. In this work, it is treated as a free parameter since the kinematics of interest is in the nonperturbative region.

At this step, one can carry out the integration over the azimuthal angles of $r_{\perp}$ and $b_{\perp}$. The direct production amplitude is rewritten as

$$
\begin{aligned}
\mathcal{M}_{d}= & i \zeta(1-\zeta) \cos \left(2 \phi_{\Delta}+\phi_{k}-3 \phi_{P}\right) \mathcal{E}\left(x_{g}, \Delta_{\perp}\right) \\
& +i \zeta(1-\zeta) \cos \left(\phi_{k}-\phi_{P}\right) \mathcal{A}_{d}\left(x_{g}, \Delta_{\perp}\right)+\cdots,
\end{aligned}
$$

where we only keep the angular structures which contribute to the azimuthal-independent interference cross section and the $\cos 4 \phi$ azimuthal modulation. $\phi_{k}, \phi_{P}$, and $\phi_{\Delta}$ represent the azimuthal angles of different transverse momenta, respectively. $\mathcal{E}\left(x_{g}, \Delta_{\perp}\right)$ and $\mathcal{A}_{d}\left(x_{g}, \Delta_{\perp}\right)$ are given by

$$
\begin{aligned}
\mathcal{E}\left(x_{g}, \Delta_{\perp}\right)=- & \frac{e e_{q}}{2} N_{c} \mathcal{C} E e^{i \delta \phi} \int \frac{d b_{\perp}^{2} d r_{\perp}^{2}}{4} \frac{Q_{s}^{2} r_{\perp}^{2}}{4} e^{-\frac{Q_{s}^{2} r_{\perp}^{2}}{4}} \\
& \times J_{2}\left(\left|\Delta_{\perp}\right|\left|b_{\perp}\right|\right) J_{3}\left(\left|r_{\perp}\right|\left|P_{\perp}\right|\right) \frac{\partial K_{0}\left(\left|r_{\perp}\right| e_{f}\right),}{\partial\left|r_{\perp}\right|}
\end{aligned}
$$

$$
\begin{aligned}
\mathcal{A}_{d}\left(x_{g}, \Delta_{\perp}\right)= & -e e_{q} N_{c} \mathcal{C} \int \frac{d b_{\perp}^{2} d r_{\perp}^{2}}{4}\left(1-e^{-\frac{Q_{s}^{2} r_{\perp}^{2}}{4}}\right) \\
& \times J_{0}\left(\left|\Delta_{\perp}\right|\left|b_{\perp}\right|\right) J_{1}\left(\left|r_{\perp}\right|\left|P_{\perp}\right|\right) \frac{\partial K_{0}\left(\left|r_{\perp}\right| e_{f}\right),}{\partial\left|r_{\perp}\right|}
\end{aligned}
$$

where $\mathcal{A}_{d}$ contributes to the polarization-independent interference cross section, which will be compared with STAR data to fix the constant $\mathcal{C}$. As there is no experimental evidence suggesting that the azimuthal-dependent amplitude must be real, we insert a phase on the right-hand side of Eq. (17). To have a maximal asymmetry, we assume $\delta \phi=\pi / 2$ in the following numerical calculation.

Since the two incoming nuclei take turns to act as the coherent photon source and the target, one should sum over these two possibilities on the amplitude level. The observed diffractive pattern at low transverse momentum crucially depends on such double-slit interference effect [40,41,64-66]. We noticed that the similar double-slit interference effect has been discovered in spin space [67,68]. Following the method outlined in Ref. [40], we also include the double-slit interference effect in the calculation of the interference cross section from direct production and $\rho$ decay production, the azimuthaldependent part of which is given by

$$
\begin{aligned}
\frac{d \sigma_{I}}{d \mathcal{P} . \mathcal{S} .}= & \frac{\zeta(1-\zeta) M_{\rho} \Gamma_{\rho}\left|P_{\perp}\right| f_{\rho \pi \pi}}{2(2 \pi)^{7}\left(\left(Q^{2}-M_{\rho}^{2}\right)^{2}+M_{\rho}^{2} \Gamma_{\rho}^{2}\right)} \int d^{2} \Delta_{\perp} d^{2} k_{\perp} d^{2} k_{\perp}^{\prime} \delta^{2}\left(k_{\perp}+\Delta_{\perp}-q_{\perp}\right) \cos \left(3 \phi_{P}-\phi_{k}-2 \phi_{\Delta}\right) \cos \left(\phi_{P}-\phi_{k^{\prime}}\right) \\
& \times\left\{e^{i \tilde{b}_{\perp} \cdot\left(k_{\perp}^{\prime}-k_{\perp}\right)} \mathcal{A}^{*}\left(x_{2}, \Delta_{\perp}{ }^{\prime}\right) \mathcal{E}\left(x_{2}, \Delta_{\perp}\right) \mathcal{F}\left(x_{1}, k_{\perp}\right) \mathcal{F}\left(x_{1}, k_{\perp}^{\prime}\right)+e^{i \tilde{b}_{\perp} \cdot\left(\Delta_{\perp}^{\prime}-\Delta_{\perp}\right)} \mathcal{A}^{*}\left(x_{1}, \Delta_{\perp}{ }^{\prime}\right) \mathcal{E}\left(x_{1}, \Delta_{\perp}\right) \mathcal{F}\left(x_{2}, k_{\perp}\right) \mathcal{F}\left(x_{2}, k_{\perp}^{\prime}\right)\right. \\
& \left.+e^{i \tilde{b}_{\perp} \cdot\left(\Delta_{\perp}^{\prime}-k_{\perp}\right)} \mathcal{A}^{*}\left(x_{2}, \Delta_{\perp}{ }^{\prime}\right) \mathcal{E}\left(x_{1}, \Delta_{\perp}\right) \mathcal{F}\left(x_{1}, k_{\perp}\right) \mathcal{F}\left(x_{2}, k_{\perp}^{\prime}\right)+e^{i \tilde{b}_{\perp} \cdot\left(k_{\perp}^{\prime}-\Delta_{\perp}{ }^{\prime}\right)} \mathcal{A}^{*}\left(x_{1}, \Delta_{\perp}{ }^{\prime}\right) \mathcal{E}\left(x_{2}, \Delta_{\perp}\right) \mathcal{F}\left(x_{2}, k_{\perp}\right) \mathcal{F}\left(x_{1}, k_{\perp}^{\prime}\right)\right\} \\
& + \text { c.c. },
\end{aligned}
$$


where $d \mathcal{P} . \mathcal{S}$. $=d^{2} p_{1 \perp} d^{2} p_{2 \perp} d y_{1} d y_{2} d^{2} \tilde{b}_{\perp} \cdot y_{1}$ and $y_{2}$ are the final-state pions' rapidities. $k_{\perp}, \Delta_{\perp}, k_{\perp}$,' and $\Delta_{\perp}^{\prime}$ are the incoming photon's transverse momenta and nucleus recoil transverse momenta in the amplitude and the conjugate amplitude, respectively. $\tilde{b}_{\perp}$ is the transverse distance between the center of the two colliding nuclei. $\mathcal{F}\left(x, k_{\perp}\right)$ describes the probability amplitude for finding a photon carries certain momentum. The squared $\mathcal{F}\left(x, k_{\perp}\right)$ is just the standard photon TMD distribution $f\left(x, k_{\perp}\right)$. Using the equivalent photon approximation, xone has

$$
\mathcal{F}\left(x, k_{\perp}\right)=\frac{Z \sqrt{\alpha_{e}}}{\pi}\left|k_{\perp}\right| \frac{F\left(k_{\perp}^{2}+x^{2} M_{p}^{2}\right)}{\left(k_{\perp}^{2}+x^{2} M_{p}^{2}\right)}
$$

where $x$ is constrained according to $x=$ $\sqrt{\frac{P_{\perp}^{2}+m_{\pi}^{2}}{S}}\left(e^{y_{1}}+e^{y_{2}}\right) \cdot M_{p}$ is proton mass, $Z$ is the nuclear charge number, and $F$ is the nuclear charge form factor parametrized with the standard Woods-Saxon distribution. Note that the incoming photon carries the different transverse momenta in the amplitude and the conjugate amplitude since we fixed $\tilde{b}_{\perp}[56,69-73]$.

The emergence of these nontrivial azimuthal correlations can be intuitively understood as follows: the linear polarization photon state is the superposition of different helicity states. As illustrated in Fig. 1, the incoming photon carries 1 unit spin angular momentum in the amplitude while it carries -1 unit spin angular momentum in the conjugate amplitude. The orbital angular momentum transferred to quark pair via the two gluon exchange is 2 unit angular momentum as far as the elliptic gluon distribution is concerned. As a result, the orbital angular momentum carried by the produced dipion system is 3 unit in the amplitude and -1 in the conjugate amplitude, correspondingly. Such interference amplitude leads to a $\cos 4 \phi$ angular modulation. If one averages over coherent photon polarization, this mechanism gives rise to $\cos 2 \phi$ asymmetry as well, though the dominant contribution to it comes from different source [40].

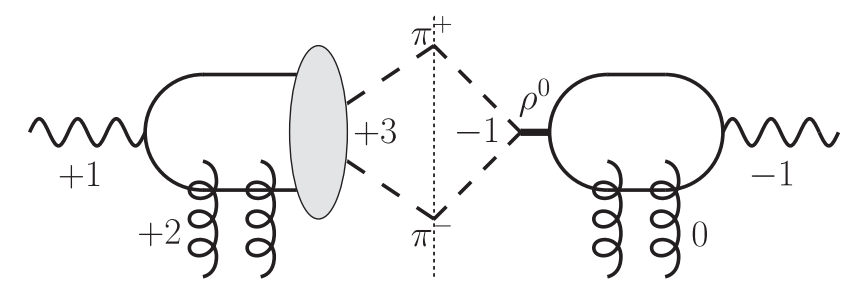

FIG. 1. $\cos 4 \phi$ azimuthal asymmetry results from the interference between the $p$ wave and the $f$ wave of pion pairs that are from the decay of $\rho^{0}$ meson in conjugate amplitude, and are from direct production in the amplitude. The color-neutral exchange in the amplitude described by the elliptic gluon distribution effectively carries 2 unit orbital angular momentum. The incident photon is linearly polarized.

\section{NUMERICAL ESTIMATIONS}

We now introduce models/parametrizations that are necessary for numerical calculations. First of all, the dipole-nucleus scattering amplitude (the azimuthal independent part) is parametrized in terms of dipole-nucleon scattering amplitude $\mathcal{N}\left(r_{\perp}\right)$ [74-78],

$$
N\left(b_{\perp}, r_{\perp}\right) \approx 1-\left[1-2 \pi B_{p} T_{A}\left(b_{\perp}\right) \mathcal{N}\left(r_{\perp}\right)\right]^{A},
$$

where we adopt the Golec-Biernat-Wusthoff model for $\mathcal{N}\left(r_{\perp}\right)$. We also made the numerical estimates with a more sophisticated treatment for $\mathcal{N}\left(r_{\perp}\right)$ [76-79], which leads to the similar results. The nuclear thickness function $T_{A}\left(b_{\perp}\right)$ is determined with the Woods-Saxon distribution in our numerical calculation, and $B_{p}=4 \mathrm{GeV}^{-1}$. For the scalar part of vector meson function, we use the "GaussLC" wave function also taken from Refs. [74,75]: $\Omega^{*}\left(\left|r_{\perp}\right|, z\right)=\beta z(1-z) \exp \left[-\frac{r_{\perp}^{2}}{2 R_{\perp}^{2}}\right]$, with $\beta=4.47, R_{\perp}^{2}=$ $21.9 \mathrm{GeV}^{-2}$. The nuclear thickness function is estimated with the Woods-Saxon distribution, $F\left(\vec{k}^{2}\right)=$ $\int d^{3} r e^{i \vec{k} \cdot \vec{r}} \frac{C^{0}}{1+\exp \left[\left(r-R_{W S}\right) / d\right]}$, where $R_{W S}(\mathrm{Au}: 6.38 \mathrm{fm})$ is the radius and $\mathrm{d}$ (Au.: $0.535 \mathrm{fm})$ is the skin depth. $C^{0}$ is the normalization factor.

UPCs events measured at RHIC are triggered by detecting accompanied forward neutron emissions. The impact parameter dependence of the probability for emitting any number of neutrons from an excited nucleus (referred to as the " $X_{n}$ " event) is described by the function $P\left(\tilde{b}_{\perp}\right)=1-$ $\exp \left[-P_{1 n}\left(\tilde{b}_{\perp}\right)\right]$ with $P_{1 n}\left(\tilde{b}_{\perp}\right)=5.45 \times 10^{-5} \frac{Z^{3}(A-Z)}{A^{2 / 3} \tilde{b}_{\perp}^{2}} \mathrm{fm}^{2}$. Therefore, the "tagged" UPC cross section is defined as

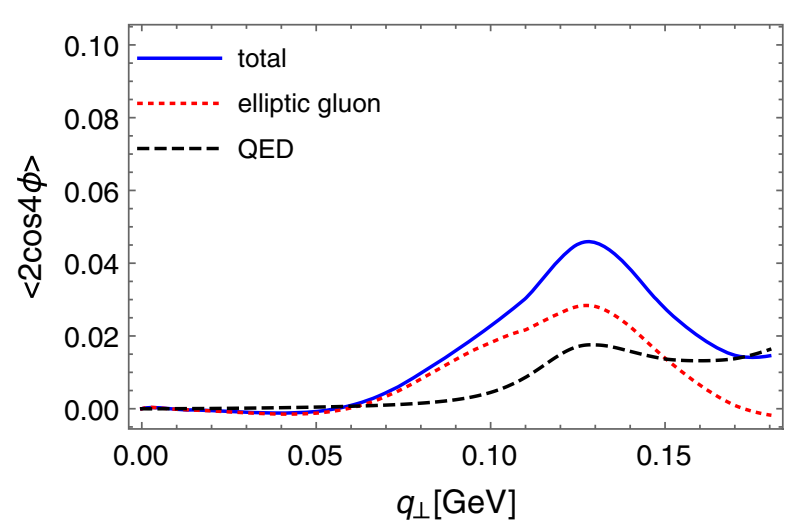

FIG. 2. The asymmetry is plotted as the function of $q_{\perp}$ for RHIC energy $\sqrt{S}=200 \mathrm{GeV}$. The rapidities $y_{1}, y_{2}$ of produced pions are integrated over the region $[-1,1]$ and $Q$ is integrated over the region $[0.6 \mathrm{GeV}$ and $1 \mathrm{GeV}]$. The contributions from the final-state soft photon radiation and elliptic gluon distribution to the asymmetry are shown separately. 


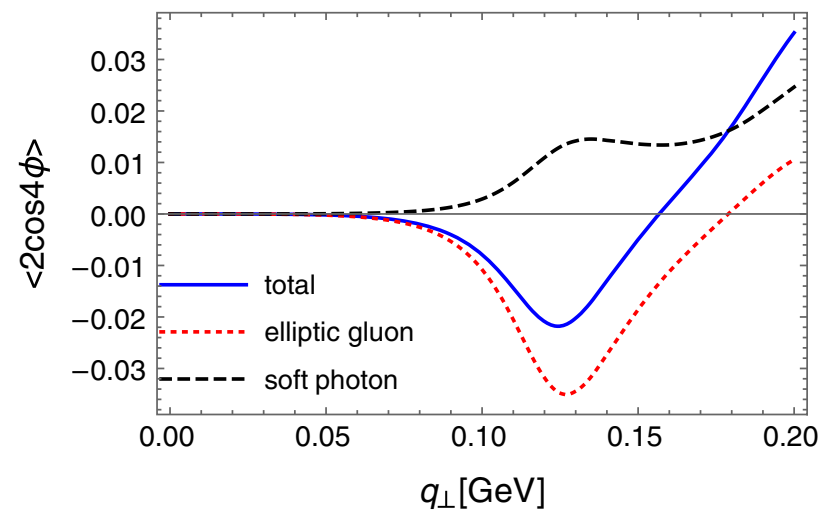

FIG. 3. The asymmetry in photon production of dipion in eA collisions at EIC is plotted as the function of $q_{\perp}$ for the center of mass energy $\sqrt{S}=100 \mathrm{GeV}$. The rapidities $y_{1}, y_{2}$ of produced pions are integrated over the region [2,3] and the invariant mass of dipion $Q$ is integrated over the region [0.6 GeV and $1 \mathrm{GeV}$ ]. Transverse momentum carried by the quasireal photon emitted from electron beam is required to be smaller than $0.1 \mathrm{GeV}$.

$$
2 \pi \int_{2 R_{A}}^{\infty} \tilde{b}_{\perp} d \tilde{b}_{\perp} P^{2}\left(\tilde{b}_{\perp}\right) d \sigma\left(\tilde{b}_{\perp}, \ldots\right)
$$

With all these ingredients, we are ready to perform numerical study of the $\cos 4 \phi$ azimuthal asymmetry for RHIC kinematics.

We first compute the azimuthal averaged cross section and compare it with STAR data to fix the coefficient $\mathcal{C} \approx-10$, which determines the relative magnitude between the direct pion pair production and that via $\rho^{0}$ decay. We then are able to compute the $\cos 4 \phi$ asymmetry from the elliptic gluon distribution. The QED and the elliptic gluon distribution contributions to the asymmetry are separately presented in Fig. 2. If we only take into account the final-state soft photon radiation effect, the theory calculation severely underestimates the experimental data. To match the STAR data [39], a rather large value of the coefficient $E=0.4$ in Eq. (15))which is roughly one order of magnitude larger than the perturbative estimate for $E[10,17]$, has been used in our numerical calculation. Since we are dealing with the deep nonperturbative region, it is hard to tell whether such large value for $E$ is reasonable or not. Moreover, there are many uncertainties associated with the transition from quark pair to dipion. Another nonperturbative model for describing this transition might lead to a much larger asymmetry with the same value of $E$. Nevertheless, as demonstrated in Fig. 2, it is clear that the elliptic gluon distribution is a necessary element to account for the observed asymmetry (around 10\%).

We also compute the $\cos 4 \phi$ azimuthal asymmetry in the process $\gamma+A \rightarrow A^{\prime}+\pi^{+}+\pi^{-}$for EIC kinematics with the same set parameters. It is shown in Fig. 3 that the contribution from the elliptic gluon distribution to the asymmetry flips the sign as the result of the absence of the double-slit interference effect in electron-nucleus (eA) collisions. It would be very interesting to test this predication at the future EIC. In view of the recent findings $[23,24]$, this might be the only clean observable to probe the gluon Wigner function at EIC, because it is free from the contamination due to the final-state soft gluon radiation effect.

\section{CONCLUSION}

We studied $\cos 4 \phi$ azimuthal asymmetry in exclusive dipion production near $\rho^{0}$ resonance peak in UPCs. Both the final-state soft photon radiation effect and the elliptic gluon distribution can give rise to such a asymmetry. It is shown that the QED effect alone, which can be cleanly computed, is not adequate to describe the STAR data. On the other hand, with some model-dependent input, a better agreement with the preliminary STAR data is reached after including the elliptic gluon distribution contribution, though the theory calculation still underestimates the measured asymmetry. This thus leads us to conclude that the observed $\cos 4 \phi$ asymmetry might signal the very existence of the nontrivial quantum correlation encoded in elliptic gluon distribution.

\section{ACKNOWLEDGMENTS}

We thank Raju Venugopalan, Zhang-bu Xu, James Daniel Brandenburg, Feng Yuan, and Chi Yang for valuable discussions. J.Z. has been supported by the National Natural Science Foundation of China under Grant No. 11675093. Y.Z. has been supported by the Natural Science Foundation of Shandong Province under Grant No. ZR2020MA098. C.Z. is supported by the China Postdoctoral Science Foundation under Grant No. 2019M662317.
[1] A. V. Belitsky, X.-d. Ji, and F. Yuan, Phys. Rev. D 69, 074014 (2004).

[2] C. Lorce and B. Pasquini, Phys. Rev. D 84, 014015 (2011).

[3] Y. Hatta, Phys. Lett. B 708, 186 (2012).
[4] X. Ji, X. Xiong, and F. Yuan, Phys. Rev. Lett. 109, 152005 (2012).

[5] Y. Hatta, Y. Nakagawa, F. Yuan, Y. Zhao, and B. Xiao, Phys. Rev. D 95, 114032 (2017). 
[6] S. Meissner, A. Metz, and M. Schlegel, J. High Energy Phys. 08 (2009) 056.

[7] S. Meissner, A. Metz, M. Schlegel, and K. Goeke, J. High Energy Phys. 08 (2008) 038.

[8] Y. Hatta, B.-W. Xiao, and F. Yuan, Phys. Rev. Lett. 116, 202301 (2016).

[9] T. Altinoluk, N. Armesto, G. Beuf, and A. H. Rezaeian, Phys. Lett. B 758, 373 (2016).

[10] J. Zhou, Phys. Rev. D 94, 114017 (2016).

[11] Y. Hagiwara, Y. Hatta, R. Pasechnik, M. Tasevsky, and O. Teryaev, Phys. Rev. D 96, 034009 (2017).

[12] E. Iancu and A. H. Rezaeian, Phys. Rev. D 95, 094003 (2017).

[13] R. Boussarie, Y. Hatta, B.-W. Xiao, and F. Yuan, Phys. Rev. D 98, 074015 (2018).

[14] Y. Hatta, B.-W. Xiao, and F. Yuan, Phys. Rev. D 95, 114026 (2017).

[15] H. Mäntysaari, N. Mueller, and B. Schenke, Phys. Rev. D 99, 074004 (2019).

[16] H. Mäntysaari, N. Mueller, F. Salazar, and B. Schenke, Phys. Rev. Lett. 124, 112301 (2020).

[17] H. Mäntysaari, K. Roy, F. Salazar, and B. Schenke, Phys. Rev. D 103, 094026 (2021).

[18] A. Dumitru, H. Mäntysaari, R. Paatelainen, K. Roy, F. Salazar, and B. Schenke, in Proceedings of the 28th International Workshop on Deep Inelastic Scattering and Related Subjects (SciPost Fundation, Amsterdam, The Netherlands, 2021).

[19] S. Bhattacharya, A. Metz, V. K. Ojha, J.-Y. Tsai, and J. Zhou, arXiv:1802.10550.

[20] S. Bhattacharya, A. Metz, and J. Zhou, Phys. Lett. B 771, 396 (2017); 810, 135866(E) (2020).

[21] S. Catani, M. Grazzini, and A. Torre, Nucl. Phys. B890, 518 (2014).

[22] S. Catani, M. Grazzini, and H. Sargsyan, J. High Energy Phys. 06 (2017) 017.

[23] Y. Hatta, B.-W. Xiao, F. Yuan, and J. Zhou, Phys. Rev. Lett. 126, 142001 (2021).

[24] Y. Hatta, B.-W. Xiao, F. Yuan, and J. Zhou, Phys. Rev. D 104, 054037 (2021).

[25] CMS Collaboration, Report No. CMS-PAS-HIN-18-011, 2020.

[26] G. Baur, K. Hencken, and D. Trautmann, J. Phys. G 24, 1657 (1998).

[27] S. Klein and P. Steinberg, Annu. Rev. Nucl. Part. Sci. 70, 323 (2020).

[28] S. Klein et al., in Proceedings of the 2022 Snowmass Summer Study (2020) [arXiv:2009.03838].

[29] Y. Hatta, A. Rajan, and D.-L. Yang, Phys. Rev. D 100, 014032 (2019).

[30] Y. Hatta, M. Strikman, J. Xu, and F. Yuan, Phys. Lett. B 803, 135321 (2020).

[31] P. A. Steinberg (ALICE, ATLAS, CMS, LHCb, and STAR Collaborations), Nucl. Phys. A1005, 122007 (2021).

[32] K. Hattori, H. Taya, and S. Yoshida, J. High Energy Phys. 01 (2021) 093.

[33] P. Copinger and S. Pu, Int. J. Mod. Phys. A 35, 203005 (2020).

[34] M. Strikman, Nucl. Phys. B, Proc. Suppl. 179-180, 111 (2008).
[35] C. Li, J. Zhou, and Y.-J. Zhou, Phys. Lett. B 795, 576 (2019).

[36] C. Li, J. Zhou, and Y.-J. Zhou, Phys. Rev. D 101, 034015 (2020).

[37] B.-W. Xiao, F. Yuan, and J. Zhou, Phys. Rev. Lett. 125, 232301 (2020).

[38] J. Adam et al. (STAR Collaboration), Phys. Rev. Lett. 127, 052302 (2021).

[39] J. D. Brandenburg et al. (STAR Collaboration), Proceedings of the Quark Matter 2019, Wuhan, China (Elsevier, Amsterdam, The Netherlands, 2019).

[40] H. Xing, C. Zhang, J. Zhou, and Y.-J. Zhou, J. High Energy Phys. 10 (2020) 064.

[41] W. Zha, J. D. Brandenburg, L. Ruan, Z. Tang, and Z. Xu, Phys. Rev. D 103, 033007 (2021).

[42] J. D. Brandenburg, W. Zha, and Z. Xu, arXiv:2103.16623.

[43] Y. Hagiwara, C. Zhang, J. Zhou, and Y.-J. Zhou, Phys. Rev. D 103, 074013 (2021).

[44] P. Soding, Phys. Lett. 19, 702 (1966).

[45] M. Klusek-Gawenda and A. Szczurek, Phys. Rev. C 87, 054908 (2013).

[46] A. Bolz, C. Ewerz, M. Maniatis, O. Nachtmann, M. Sauter, and A. Schöning, J. High Energy Phys. 01 (2015) 151.

[47] P. Hägler, B. Pire, L. Szymanowski, and O. V. Teryaev, Phys. Lett. B 535, 117 (2002); 540, 324(E) (2002).

[48] P. Hagler, B. Pire, L. Szymanowski, and O. V. Teryaev, Eur. Phys. J. C 26, 261 (2002).

[49] L. Adamczyk et al. (STAR Collaboration), Phys. Rev. C 96, 054904 (2017).

[50] A. M. Sirunyan et al. (CMS Collaboration), Eur. Phys. J. C 79, 702 (2019).

[51] S. Acharya et al. (ALICE Collaboration), J. High Energy Phys. 06 (2020) 035.

[52] M. Ryskin, Z. Phys. C 57, 89 (1993).

[53] S. J. Brodsky, L. Frankfurt, J. Gunion, A. H. Mueller, and M. Strikman, Phys. Rev. D 50, 3134 (1994).

[54] J. Bartels, K. J. Golec-Biernat, and K. Peters, Acta Phys. Pol. B 34, 3051 (2003).

[55] Y. Hagiwara, Y. Hatta, R. Pasechnik, and J. Zhou, Eur. Phys. J. C 80, 427 (2020).

[56] S. Klein, A. Mueller, B.-W. Xiao, and F. Yuan, Phys. Rev. Lett. 122, 132301 (2019).

[57] M. Diehl, T. Gousset, B. Pire, and O. Teryaev, Phys. Rev. Lett. 81, 1782 (1998).

[58] M. V. Polyakov, Nucl. Phys. B555, 231 (1999).

[59] M. Diehl, T. Gousset, and B. Pire, Phys. Rev. D 62, 073014 (2000).

[60] B. Pire and L. Szymanowski, Phys. Lett. B 556, 129 (2003).

[61] M. Diehl, Phys. Rep. 388, 41 (2003).

[62] L. D. McLerran and R. Venugopalan, Phys. Rev. D 49, 3352 (1994).

[63] L. D. McLerran and R. Venugopalan, Phys. Rev. D 49, 2233 (1994).

[64] S. R. Klein and J. Nystrand, Phys. Rev. Lett. 84, 2330 (2000).

[65] B. Abelev et al. (STAR Collaboration), Phys. Rev. Lett. 102, 112301 (2009).

[66] W. Zha, L. Ruan, Z. Tang, Z. Xu, and S. Yang, Phys. Rev. C 99, 061901 (2019). 
[67] H. Chen, I. Moult, and H. X. Zhu, Phys. Rev. Lett. 126, 112003 (2021).

[68] H. Chen, I. Moult, and H. X. Zhu, arXiv:2104.00009.

[69] M. Vidovic, M. Greiner, C. Best, and G. Soff, Phys. Rev. C 47, 2308 (1993).

[70] W. Zha, J. D. Brandenburg, Z. Tang, and Z. Xu, Phys. Lett. B 800, 135089 (2020).

[71] S. Klein, A. Mueller, B.-W. Xiao, and F. Yuan, Phys. Rev. D 102, 094013 (2020).

[72] M. Kłusek-Gawenda, W. Schäfer, and A. Szczurek, Phys. Lett. B 814, 136114 (2021).

[73] B. Wu, J. High Energy Phys. 07 (2021) 002.
[74] H. Kowalski and D. Teaney, Phys. Rev. D 68, 114005 (2003).

[75] H. Kowalski, L. Motyka, and G. Watt, Phys. Rev. D 74, 074016 (2006).

[76] A. H. Rezaeian, M. Siddikov, M. Van de Klundert, and R. Venugopalan, Phys. Rev. D 87, 034002 (2013).

[77] H. Kowalski, T. Lappi, C. Marquet, and R. Venugopalan, Phys. Rev. C 78, 045201 (2008).

[78] H. Kowalski, T. Lappi, and R. Venugopalan, Phys. Rev. Lett. 100, 022303 (2008).

[79] J. Bartels, K. J. Golec-Biernat, and H. Kowalski, Phys. Rev. D 66, 014001 (2002). 